\title{
Os Cursos de Pós-graduaçáo do Instituto Nacional de Pesquisas da Amazônia.
}

Eduardo Lleras (*)

\begin{abstract}
Resumo
Os Cursos de Pós-Graduação do INPA, com sete anos de atuação na Amazônia, têm produzido até o presente mais de 150 trabalhos científicos e formado 54 Miestres e 4 Doutores em Ciências Biológicas, nas áreas de Botânica, Ecologia, Entomologia e Biologia de Água Doce e Pesca Interior. Em 1980 foi iniciado um quinto curso em Manejo Florestal; durante o mesmo ano foi iniciado o processo de re-estruturação dos Cursos. Em janeiro de 1981 entrou em vigor o novo regimento.
\end{abstract}

\section{HISTÓRICO}

Historicamente os cursos de pós-graduação foram concebidos como uma resposta à alarmante carência de pessoal técnico qualificado na Amazônia, já que esta região ocupa mais de $50 \%$ do Território Nacional e possui menos de $1 \%$ dos Mestres e Doutores do País. Dentre as causas desta situação podem cıtar-se a relutância do pessoal qualificado em trasladar-se para a Amazônia, a fraqueza das instituições de ensino superior da região que na época, com a possível exceção da Universidade Federal do Pará, não apresentavam condições condizentes ao início de programas de pós-graduação e à grande proliferação de programas de pós-graduação no resto do país (mais de 900 cursos em 1979, com mais de 35.000 alunos matriculados versus 7 cursos com 179 alunos na Amazônia para o mesmo ano)

Era então desejável aproveitar a existencia de núcleos de pessoal altamente qualificado na região para tentar, pelo menos em parte, aliviar a falta de pessoal qualificado da região através de cursos de pós-graduação em torno destes núcleos.

Existia, e ainda existe, um núcleo de pessoal altamente qualificado que poderia ser aproveitado neste sentido: o instituto Nacıonal de Pesquisas da Amazônia (INPA) .
A filosofia que levou à criação do Curso de Pós-graduação do INPA foi a seguinte: 1) é difícil ou quase impossivel atrair pessoal nacional qualificado para trabalhar na Amazônia; 2) o INPA pode e deve ter uma ativa participação na elevação do nível das Instituições de Ensino da Região, pelo menos até o momento em que estas estejam em condições de proauzir pessoal altamente qualificado e possuam a massa crítica própria para fazê-lo; 3) o INPA tem condições de atrair professores de alto nível durante períodos curtos (até 2 anos) de tempo.

De acordo com a filosofia de criação do Curso exposta anteriormente, o INPA fez convênio com a Universidade do Amazonas e assumiu a responsabilidade de montar um Curso de Pós-Graduação, não com o intuito de aproveitar as condições das diversas Universidades da região e sim com o objetivo de fortalecer essas instituições, formando pessoal altamente qualificado para o quadro de docentes das mesmas e ao mesmo tempo, treinar pessoal para seu próprio quadro de pesquisas.

A meados de 1971, o Dr. Paulo de Almeida Machado, então Diretor do INPA, convidou o Dr. Ghillean Tolmie France para organizar um curso de pós-graduação em nível de Mestrado em Botânica Tropical. O curso foi concebido como uma resposta à alarmante carência de pessoal técnico na Amazônia.

Em julho de 1973, após mais de um ano de intenso trabalho de organização foi instalado o curso de Mestrado em Botânica com doze alunos e um corpo docente excepcionalmente idôneo, já que incluia alguns cientistas de maior destaque nas suas respectivas áreas tanto do Brasil como do exterior e, dentre os quais, podem citar-se os Drs. Theodozius Dobzansky, Fritz Ehrendorfer, Jean Lagenheim, Kiaus Kubitzki, Antonio Celso Magalhães, Robert Goodland e Rolla Tryon.

(*) - Instituto Nacional de Pesquisas da Amazônia. Manaus.

SUPL. ACTA AMAZONICA 11(1): 117-123, 1981 
O sucesso deste primeiro curso foi tão grande que em 1975, o Dr. Warwick Estevam Kerr, na época Diretor do INPA, e o Magnífico Reitor da Universidade do Amazonas, Dr. Aderson Pereira Dutra, assinaram convênio que previa, entre outras coisas, a criação de cursos de pós-graduação nas áreas de Ecologia, Entomologia e Biologia de Água Doce e Pesca Interior, iniciando-se as atividades em janeiro de 1976

Em 1979, o Dr. Enéas Salati, então Diretor do INPA, autorizou a criação de especialização em Manejo Florestal dentro do Curso de Pósgraduação em Botânica, com vistas, em um futuro próximo, de criar um Curso de Manejo Florestal em nível também de Pós-graduação visto a grande importância deste para a região. Com apoio econômico da Superintendência da Zona Franca de Manaus (SUFRAMA), esta especialização teve inicio em março de 1980 com 8 alunos.

Para estruturar este curso e colaborar na reestruturação do regimento dos cursos e na reorganização dos mesmos, o Dr. Salati providenciou a vinda, em julho de 1980, dos Drs. Paulo Friedirich Bührnheim e Jesus Mardem dos Santos.

Após 6 meses de intenso trabalho, em janeiro de 1981, após aprovação pela Comissão de Pós-Graduação Integrada INPA/FUA, pas- sou a vigorar o novo regimento. Atualmente os cursos estão na fase final de reestruturação, passando de um sistema no qual as matérias eram oferecidas em regime intensivo de um mês, a um sistema semestral com 2 periodos intensivos intercalados entre os semestres.

Além de cumprir com seu objetivo básico, a pós-graduação do INPA tem gerado um grande número de pesquisas de alto nível, destacando-se como o maior produtor de trabalhos monográficos extensos e de alta qualidade (ver Tabela i). A pesquisa feita pelos alunos de pós-graduação é certamente a menos onerosa e sua qualidade por sua própria natureza, é a mais rigidamente examinada. Atualmente os estudantes de pós-graduação constituem aproximadamente uma terça parte do pessoal científico atuante do INPA.

A tabela 1 apresenta uma sinopse do curso de pós-graduação desde 1974 até o presente. Como pode ser observado, em quase 8 anos de funcionamento, além de ter produzido 54 Mes. tres e 4 Doutores, incluindo Teses e Disserta. ções, foram publicados além de 150 trabalhos científicos, trabalhos estes que são apresentados quanto às suas descobertas nas memórias dos respectivos Departamentos de Pesquisas.

TABELA 1 - Posição do Curso de Pós-Graduação até $31 / 03 / 81$.

\begin{tabular}{|c|c|c|c|c|c|c|c|c|c|c|}
\hline & & 1974 & 1975 & 1976 & 1977 & 1978 & 1979 & 1980 & 1981 & TOTAL \\
\hline ALUNOS NOVOS & $\begin{array}{l}\text { MATRICULADOS NO CURSO/ } \\
\text { MESTRADO }\end{array}$ & 12 & 0 & 39 & 0 & 39 & 0 & 38 & 0 & 128 \\
\hline ALUNOS NOVOS & $\begin{array}{l}\text { MATRICULADOS NO CURSO/ } \\
\text { DOUTORADO }\end{array}$ & 0 & 0 & 11 & 0 & 13 & 5 & 4 & 0 & 33 \\
\hline \multicolumn{2}{|c|}{ PESSOAL FORMADO, MESTRES } & 0 & 6 & 5 & 2 & 16 & 14 & 6 & 5 & 54 \\
\hline \multicolumn{2}{|c|}{ PESSOAL FORMADO, DOUTORES } & 0 & 0 & 0 & 0 & 0 & 0 & 3 & 1 & 04 \\
\hline \multicolumn{2}{|c|}{ TESES PUBLICADAS } & 0 & 0 & 3 & 6 & 3 & 5 & 6 & 0 & 23 \\
\hline \multicolumn{2}{|l|}{ TESES NO PRELO } & 0 & 0 & 0 & 0 & 0 & 0 & 0 & 35 & 35 \\
\hline \multicolumn{2}{|c|}{ OUTROS TRABALHOS PUBLICADOS } & 5 & 12 & 22 & 25 & 22 & 8 & 16 & 2 & 112 \\
\hline \multicolumn{2}{|c|}{ TRABALHO NO PRELO } & 0 & 0 & 0 & 0 & 0 & 0 & 0 & 17 & 17 \\
\hline \multicolumn{2}{|c|}{ LIVROS PUBLICADOS } & 0 & 2 & 0 & 1 & 0 & 2 & 0 & 0 & 06 \\
\hline
\end{tabular}


A Tabela 2 apresenta os pareceres do Conselho Federal de Educação nos quais foram credenciados os diversos cursos. Pode obser. var-se que 4 cursos estão credenciados a nível de Mestrado e 2 a nível de Doutourado. Em futuro próximo serão encaminhados processos de credenciamento para Doutourado em Ecolo. gia e Biologia de Água Doce e Pesca Interior e será iniciado o processo de recredenciamento do Mestrado em Botânica que cumpre este ano 5 anos de credenciamento.

TABELA 2 - Pareceres do Conselho Federal de Educação credenciando os diversos cursos de Pós-Graduação dio INPA/FUA.

\begin{tabular}{|c|c|c|c|}
\hline PARECER CFE & CURSO DE PÓS-GRADUAÇĀO EM & NIVEL & TITULO \\
\hline $1546 / 76$ & Ciências Biológicas (Botânica) & Mestrado & $\begin{array}{l}\text { Mestre em Ciências Biológicas, } \\
\text { área de Botânica }\end{array}$ \\
\hline $7296 / 78$ & $\begin{array}{l}\text { Biologia, área de concentração em } \\
\text { Biologia de Água Doce e Pesca } \\
\text { Interior }\end{array}$ & Mestrado & $\begin{array}{l}\text { Mestre em Biologia, área de } \\
\text { Água Doce e Pesca Interior }\end{array}$ \\
\hline $7297 / 78$ & $\begin{array}{l}\text { Biologia, com área de concentra- } \\
\text { ção em Ecologia }\end{array}$ & Mestrado & $\begin{array}{l}\text { Mestre em Biologia, área de } \\
\text { Ecologia }\end{array}$ \\
\hline \multirow[t]{2}{*}{$1513 / 79$} & $\begin{array}{l}\text { Ciências Biológicas, área de con- } \\
\text { centração em Entomologia }\end{array}$ & Mestrado & $\begin{array}{l}\text { Mestre em Ciências Biológicas, } \\
\text { área de Entomologia }\end{array}$ \\
\hline & & Doutorado & $\begin{array}{l}\text { Doutor em Ciências, área de } \\
\text { Entomologia }\end{array}$ \\
\hline $1619 / 79$ & Ciências Biológicas (Botânica) & Doutorado & $\begin{array}{l}\text { Doutor em Ciências, área de } \\
\text { Botânica. }\end{array}$ \\
\hline
\end{tabular}

\section{PERSPECTIVAS}

Além dos cursos atualmente em funcionamento, Botânica, Ecologia, Entomologia, Biologia de Água Doce e Pesca interior e Manejo Florestal, os quais continuarão funcionando em prazo indeterminado seja dentro do convênio INPA/FUA, seja entregues à responsabilidade da Universidade do Amazonas, faz-se prioritária a implantação de outros cursos ou áreas de pós-graduação de importância para a Amazônia.

(a) QUímicA DE PRODUTOS NATURAIS atualmente está sendo estudada a possibilidade de implantação deste curso dentro do convênio INPA/FUA; existe pessoal qualificado para formação do corpo docente, sendo que năo será necessário a vinda de professores visitantes. O curriculum do mesmo está sendo estudado e considera-se factível iniciar atividades em janeiro de 1982. Esta especialização é considerada prioritária face, entre outras coisas, à necessidade inadiável de se obter recursos naturais renováveis que possam substituir
- petróleo tanto como combustível quanto como materia-prima para indústrias tais como piásticos. Há grande interesse por parte da SUDHEVEA na implantação de pós-graduação nesta área no INPA.

(b) SOLOS TROPICAIS - como no caso anterior, este curso é de prioridade tanto regional quanto nacional já que o conhecimento detalhado dos solos da Amazônia e estudos sobre seu manejo e aproveitamento são essenciais para o desenvolvimento racional da região. $\mathrm{Na}$ região existem os recursos humanos necessários para formar o núcleo deste curso.

(c) NUTRIÇÃo - a formação de núcleos regionais de pesquisas e pós-graduação em nutrição é um dos objetivos prioritários atuais. O INPA, atualmente. é o órgão regiona! que possui o maior e mais dinâmico grupo de nutrição; por outro lado, a região com suas características especiais apresenta problemas nutricionais que mal podem ser resolvidos em base a pesquisas realizadas em outras partes 
do pais, pesquisas estas que muitas vezes levam a soluções inaplicáveis na Amazônia. O intuito atual é de fortalecer este grupo dando-Ihe condições para assumir um programa de treinamento a alto nivel.

Pode dizer-se que os três cursos acima citados são programas para implantação a curto prazo, sendo que certamente outros virão a ser necessários a médio e longo prazo. Ainda se se tem postulado que o INPA é um Instituto de Pesquisas e não de Ensino, a experiência de outros países e de outros centros no país indica que a pesquisa não pode manter-se desligada do ensino e ser realmente dinâmica. Dentre as missỏes do INPA na Amazônia, está o treinamento de pesscal para pesquisa e ensino, fornecendo pessoal altamente qualificado para os quadros de pesquisa de outras instituições da região. Eventualmente, uma vez que as universidades atinjam massas criticas suficientes para poder assumir a responsabilidade de treinamento a nivel de pós-graduação, o INPA poderá passar o encargo às mesmas atuando apenas no ambito da parte de pesquisa do treinamento. Por enquanto deverá continuar assumindo de maneira giobal o mesmo.

O problema da Educação em geral na Amazônia é um problema complexo em todos os niveis (Lleras, 1979). No ambito da pós-graduação é ainda mais crítico que em qualquer nutro nível já que os centros geradores de pós graduandos estão na sua grande maioria situados fore da Amazônia Legal e os produtos destes centros são em alto grau inacessíveis para a região. Com mais de 900 cursos de pós-graduação no restante do país e com uma população estudantil a nível de pós-graduação de mais de 35000 estudantes (Della Senta, 1977), a desproporção entre o resta do Brasil e a Amazônia, que no mesmo ano possuia 7 cursos com menos de 150 estudantes, é alarmante, já que não há uma tendência para que o superhabit de mestres e doutoures migrem para a Amazônia. Schwartzman (1979) discutirino a excessiva proliferação de pós-graduação no Brasil sugere uma concentração de recursos eliminando cursos e argumentando que a pósgraduação é uma medida de excelência que não pode ser democráticamente distribuída por todo o território nacional.

Porém, os mecanismos burocráticos de determinação de excelências estão distribuídos em dois ou três estados que determinam os padrões para o país. Face a relutância do pessoal destes mesmos estados em aceitar migrar para as áreas em desenvolvimento, tais como a Amazônia, estas áreas têm por força de necessidade que continuar a desenvolver um agressivo e dinâmico programa de pós-graduação. Sendo que o INPA é um dos centros que possui maior massa critica em uma grande gama de áreas, não pode deixar de cumprir com esta missăo.

\section{DISSERTAÇÕES DE MESTRADO DEFENDIDAS NO INPA}

01. Pedro Luiz Braga Lisboa - Aspectos Ecológicos de Glycoxylon inophyllum (Mart., ex Miq.) Ducke (Sapotaceae) / 28.08.75.

02. Regina Célia Lobato Lisboa - Brioecologia de uma Campina Amazônica / 28.08.75.

03. Marlene Freitas da Silva - Revisão Taxonômica do Gênero Peltogine Vog. (Leguminosae-Caesalpinioideae) / 29.08.75.

04. Pedro Nonato da Conceição - Alguns Aspectos Ecofisiológicos de Floresta Húmida de Terra Firme / 18.11.75.

05. Léa Maria Medeiros Carreira - Morfologia Polínica de Plantas Lenhosas de Campina / 25.11.75.
06. Miramy Macedo - Dispersão de Plantas Lenhosas de uma Campina Amazônica / 25.11. 75 .

07. Byron W. P. de Albuquerque - Revisão Taxonômica das Rutaceae do Estado do Amazonas / 28.01.76.

08. Pedro Ivo Soares Braga - Aspectos Bíológicos das Orquidaceae de uma Campina da Amazônia Central / 28.01.76.

09. Marilene Marinho N. Braga - Anatomia Foliar de Bromeliaceae da Campina / 29.01 .76 . 
10. Raimunda Conceição Q. de Vilhena Anatomia foliar de Humiriaceae / 29.01.76.

11. izonete de Jesus da S. Araújo - Número Cromossômicos de Pteridófitas de Manaus / 30.01.76.

12. Moacyr E. Medri - Aspectos da Anatomia Ecológica de Folhas de Hevea brasiliensis Muell. Arg. / 15.12.77.

13. Miguel Petrere Júnior - Pesca e Esforço de Pesca no Estado do Amazonas /21.12.77.

14. Mário Dantas - Pastagens da Amazônia Central: Ecologia e Fauna do Solo / 30.01 .78 .

15. Eloy Guillermo Castellón Bermudez - Biologia e Ecologia de Synoeca surinama L. (Hymenóptera - Vespidae) / 10.02.78.

16. José Maria Albuquerque - Identificação de Plantas Invasoras de Culturas da Região de Manaus / 07.03.78.

17. Adelmar Gomes Bandeira - Ecologia de Termitas da Amazônia Central: Efeitos do desmatamento sobre as populações e fixa. ção de Nitrogenio / 13.03.78.

18. Inocêncio de Souza Gorayeb - Detecção de Predadores Naturais das Larvas de Simulium fulvinotum Cerq. e Melo (Diptera-Nematócera) / 28.03.78.

19. Nélio Roberto dos Reis - Morcegos da Região de Manaus e suas relações com Fungos Patogênicos / 30.03.78.

20. Júlio Dellome Filho - Fatores Físicoquímicos de Simulidade (Diptera - Nematocera) / 05.05.78.

21. Carlos José Esteves Gondim - Alguns Aspectos da Biologia Reprodutiva do Guaraná (Paullinia cupana var. sorbilis (Mart.) Ducke) Sapindaceae / 15.05.78.

22. Antony Bennett Anderson - Aspectos Florísticos e Fitogeográficos de Campinas e Campinaranas na Amazônia Central / 23.05.78.

23. Ronaldo de Almeida - lluminação Solar de Interiores: Análise de Dois Sistemas / 26.05.78.

24. Norival Dagoberto Paraluppi - Alguns Aspectos da Biologia e do Comportamento do Anopheles (N) nuneztovari Gabaldon (Diptera-Culicidade) dos Arredores da Cidade de Manaus. Amazonas, Brasil. / 04.07.78.
25. Bento Melo Mascarenhas - Contribuição ao Conhecimento de Limnogonus recurvus Drake \& Harris, 1930 (Hemíptera: Gerridae) / 27.07.78.

26. Maria Gercília Mota Soares - Aspectos Ecológicos e Alimentação dos Peixes do Igarapé do Porto, Aripuanã, MT / 03.08.78.

27. Leoneza Herculano Soares - Revisão Taxonômica dos Sciaenideos de Água Doce na Região Amazônica Brasileira (Osteichthyes, Perciformes, Scianidae) / 04.08.78.

28. Germano Guarim Neto - Revisão Taxonômica das Espécies Brasileiras do Gênero Talisia Aublet (Sapindaceae) / 03.1C.78.

29. Geraldo Mendes dos Santos - Estudio da Alimentação, Reprodução e Aspectos da Sistemática de Schizodon fasciatus Agassiz, 1829, Rhytiodus microlepis Kner, 1859 e Rhytiodus argenteofuscus Kner, 1859 do Lago Janauacá, AM, Brasil (Osteichthyes, Characoidei, Ancstomidae) / 25.01.79.

30. Suely Maria La Torraca - Efeitos da Inoculação de Micorrizas VA no Crescimento de Vignia unguiculata (L.) Walp., em três solos de Terra Firme / 15.02.79.

31. Ermelinda Maria Delamonica Freire Contribuição ao Conhecimento dos Fungos Clavarioides da Amazônia / 16.02.79

32. Carolina Joana Da Silva - Considerações sobre a Biologia Reprodutiva de Pistia stratioites L. (Araceae) / 19.03.79.

33. Ricardo Augusto Pessoa Braga - Contribuição à Ecologia e Biologia de Asthenopus curtus Hagen (Insecta - Efemeroptera) dos Arredores de Manaus (Amazônia Central) / 20.03.79.

34. Neuza Massae Azakawa - Ocorrência e Características de Azospirilum spp. no solo e nas raízes de gramíneas na Amazônia Central / 08.05.79.

35. Maurício A. Mendonça - Contribuição ao Conhecimento da Biologia de Latrodectus geographicus Hasselt, 1888 (Araneae: Theridiidae) / 14.08.79.

36. Aloísio Rodrigues Ramos - Aspectos do Nicho Alimentar de Coleodactylus amazonicus (Saurea, Gekkonidae) / 29.08.79.

37. Lauro Eduardo Bacca - Peixe-Boi (Trichecus inunguis, Sirenia) no Ciclo de Nutrientes em Águas da Amazônia: Experimento de Laboratório / 25.09.79. 
38. Martha de Aguiar Falcão - Aspectos Fenológicos, Ecológicos e de Produtividade de Algumas Fruteiras Cultivadas na Amazônia / 28.09.79.

39. Míriam A. da S. Serrano - Contribuições à Sistemática de Poekiloptera Latr., 1796 (Homoptera, Flatidae) com observações da Biologia e Comportamento do Gênero / 30.10.79.

40. José Albertino Raphael - Estudo da !dade Fisiológica de quatro populações de Tabanidae (Diptera) no Campus Universitário Manaus, Brasil / 05.11.79.

41. Antonio Paulino Andrade de Luna Dias Observações sobre a Biologia e morfologia de Onchoscelis germari (Boheman, 1837) (Coleoptera, Curailionidade) Broca do Marupá / 06.11.79.

42. Francisco Martinho Carvalho - Estudo da Alimentação, Desenvolvimento dos Ovários e Composição Química de Hypophthalamus edentatus SPIX e Potamorhina pristigaster (Steindachner) / 09.11.79.

43. Joselita Maria M. dos Santos - Aspectos Biológicos e Isoensimáticos de Anopheles (N) darlingi Root, 1926 (Diptera Culicidade) / 23.11.79.

44. Lindalva Paes de Albuquerque - Contribuição A Sistemática de Taeniaptera Macquart, 1835 (Diptera, Micropezidae) com observações da Ecologia de Espécles da Amazônia Central /27.11.79.

45. Angela Maria Conte Leite - Ecologia de Plântulas de Pithecolobium racemosum Ducke / 04.02.80.

46. Ilka Maria Prazeres Paixão - Estudo da Alimentação e Reprodução de Mylossoma duriventris, Cuvier, 1818 (Pisces, Charachoidei) / 28.04.80.
47. Maria Cristina de Melo Amorozo - Alimentação em um Bairro pobre de Manaus / 24.06.80.

48. Barbara Anne Robertson - Composição, Abundância e Distribuição de Cladocera da Região de Água Livre da Represa de Curuá-Una, Pará / 20.10.80.

49. Raimunda Gonçalves de Almeida - Aspectos Taxonômicos e Hábitos Alimentares de três Espécies de Triportheus (Pices: Caracodei, Caracide) do Lago do Castanho; Amazonas / 15.12.80.

50. Maria Silvia de Mendonça - Aspectos Anatômicos e Distribuição de Vasos Laticíferos de Algumas Espécies de Manihot (Maniçobas) / 22.12.80.

51. Fanny Lianos Henriquez - Revisão Taxonômica das Espécies Brasileiras do Genero Manilkara Adanson (Sapotaceae) / 12.02.81.

52. Ceclie M. A. B. Biancardi - Aspectos Epidemiológicos da Leshmaniose Cutânea na Rodovia BR 364/, Território Federal de Rondônia / 19.02.81.

53. Julia Ignês do N. Salém José - Blastogênose em Hanseniase no Estado do Amazonas: Importância da Vitamina $A$ e do Zinco / 20.02.81.

54. Antonio Carlos Weber - Biologia Floral de Algumas Annonaceae na Região de Manaus, AM. / 30.03.81.

55. Ronaldo Borges Barthem - Considerações Sobre a Pesca Esperimental com Rede de Espera em Lagos da Amazônia Central. / 15.04.81.

\section{TESES DE DOUTOURADO DEFENDIDAS NO INPA}

01. Maria Alves de Souza - O Gênero Phellinus Quelet (Hymenochetaceae) na Amazônia Brasileira / 18.01.80.

02. Marlene Freitas da Silva - Revisão Taxonômica do Gênero Dimorphandra Schott (Leguminosae - Caesalpinioideae) .... / 21.01.80.
03. Dayse Vasquez Martins - Desmidioflórula dos Lagos Cristalino e São Sebastião, Estado do Amazonas / 21.02.80.

04. Moacyr E. Medri - Anatomia Comparada e Correlações Anatomo-Fisio-Ecológicas de seis clones de Hevea spp. / 24.02.81. 
The Graduate Courses at INPA have, in the seven years of their existence, produced more than 150 scientific publications and graduated 54 Masters and 4 Doctors in Science in Botany, Ecology, Entomology and Fresh Water Biology and Fisheries. A fifth course in Forest Managment opened in 1980; during the same year, a complete reorganization of the Courses was iniciated, with the new regulations for the same entering into effect in January of 1981.
DELLA SENTA, I.M.C.

1977 - Situação atual da Pós-Graduação Brasil-77. Brasília, MEC. DAU. CAPES.

LLERAS, $\mathrm{E}$.

1979 - Problemas populacionais e educacionais. in: Estratégias Para Política Florestal na Ama. zônia Brasileira. Acta Amazonica, 9 (4). Suplemento, 197-204.

SCHWARTZMAN, S.

1979 - Que fazer com a Pós-Graduação? Debate, set. 1979. Folheto publicado pela CAPES p. 1-3. 